\title{
Consensus in endoscopy
}

\author{
RJ Bailey (Chairman), A Barkun, J Brow, M Champion, J Deneault, R Fedorak, R Keith, E Lalor, \\ hM MacSween, N Marcon, J McHattie, A Micflikier, P Pare, R Passi, D Patel, D Petrunia, \\ E SHAFFER, J SIDOROV, S STORDY, L SUTHERLAND, R TANTON
}

\section{ETHICAL GUIDELINES FOR ENDOSCOPY}

Any discussion of medical ethics, including ethics and gastrointestinal endoscopy, must include four principles: autonomy, beneficence, nonmaleficence and justice. Autonomy is 'self-ruling'. Beneficence means kindness, to be charitable and beneficial. This special medical obligation is implicit in the doctor-patient therapeutic relationship. Nonmaleficence from an ethical view may not mean to do no harm but rather to make sure the anticipated harm or risks of therapy are worthwhile relative to the good intended from the patient's point of view. Justice requires fair treatment of patients and a respect for their rights. When reviewing the ethics of any aspect of endoscopy, it is worthwhile to keep the four principles in mind.

Endoscopists must always consider the patient's best interests, especially in today's economic times when resource allocation and utilization are restricted. Knowing that their decisions will influence the health care budget at an aggregate or provincial level, the hospital budget at an intermediate level and the patient's budget at a microlevel, endoscopists must attend to their patients' needs and requirements, while doing no harm, all in the context of limited resources.

Resources influence endoscopy. The cost per unit of endoscopy is calculable but these group goals may conflict with individual goals. Part of the financial equation is the professional's remuneration. To be paid for services rendered is morally correct and endoscopists must convince the general public of this.

Diagnostic and therapeutic safety is foremost. This implies formal training and maintenance of competence. Endoscopists must have good standards of care. Despite lack of funding and more physicians wanting to do endoscopy, training for technical and cognitive skills is mandatory. Endoscopy is not solely guiding the endoscope. After a formal training program, competence must be maintained. Short courses in endoscopy do not replace a recognized formal pro- gram. Their objectives should be clearly defined, patient safety considered and trainee competence ethically assessed.

Endoscopic research protocols must be ethical. Patients must have informed consent. Studies must be supervised and results meticulously assessed. Publication may have an impact and should be free from errors and possible misrepresentation. Informed consent is most important not only in research but for all endoscopy procedures. Patient's autonomy comes into play more in consent forms than any other aspect of endoscopy practice. Consents also must be legal; patients need to be aware of options, risk and benefits. Endoscopists should ask "What would a reasonable person do in the same circumstances?" Consents in offices are as important as those obtained in hospitals. It's best to have the consent witnessed.

All endoscopic procedures need periodic review and outcome analysis. Standards for endoscopists too need to be established. Guidelines need to apply to in-hospital as well as to free-standing endoscopy units.

\section{Ethical considerations}

- Informed consent must be secured for all procedures.

- Patients must be informed of all common risks.

- Options, risks and benefits must be discussed.

- Witness should be secured.

- Resource utilization must be studied.

- Economic restraints should not adversely influence the quality of care.

- Endoscopists must be adequately trained, no matter the specialty.

- Standards of care must be fulfilled.

This consensus document was prepared on behalf of the Canadian Association of Gastroenterology

Correspondence and reprints: Dr RJ Bailey, Hys Medical Centre, 310-11010 101 Street NW, Edmonton, Alberta T5H 4B9. Telephone 403-421-1029, fax 403-425-5533

Received for publication April 16, 1996. Accepted May 24, 1996 
TABLE 1

Recommended relative value guide

\begin{tabular}{lc}
\hline Procedure & $\begin{array}{c}\text { Relative value } \\
\text { (points) }\end{array}$ \\
\hline Gastroscopy & 1000 \\
Sigmoidoscopy & \\
$\quad$ Flexible & 500 \\
$\quad$ Rigid & 300 \\
Colonoscopy & 2500 \\
ERCP & 3000 \\
Routine endoscopic biopsy (per endoscopy) & 250 \\
ERCP biopsy and/or cytology & 400 \\
Esophageal dilation & 500 \\
\hline
\end{tabular}

ERCP Endoscopic retrograde cholangiopancreatography

\section{ECONOMICS OF ENDOSCOPY}

What is the value of endoscopy? What is the financial cost? Is the gain from endoscopy measurable? The answers to these questions are, at best, estimates in the absence of careful measurement and outcome studies.

Establishing fees in future will require identification and measurement of outcomes. Are we cost-effective practitioners? Does endoscopy benefit the patients? Is endoscopy the best alternative, and if so by how much?

Measurements will soon be the only acceptable approach and even wise advice will require documented support. Until then, with few data to answer these difficult questions, the economic value of endoscopy relative to other medical services depends on the experience of practitioners, hopefully a sage or two.

The Barer-Stoddart Report identified fee-for-service as a problem with remuneration. Provincial governments are now looking at alternative payment systems or non-fee-forservice. As well, relative value guides are being negotiated at the provincial levels for those outside an alternate payment plan.

The Canadian Association of Gastroenterology (CAG) Endoscopy Committee has reviewed provincial endoscopy fees across Canada and identified an impressive disparity from province to province and within provinces relative to a major medical consultation. Need for future negotiations and the disparities have led this professional body to consider a relative value for four major endoscopic procedures: gastroscopy, sigmoidoscopy, colonoscopy, and endoscopic retrograde cholangiopancreatography (ERCP) (Table 1).

The relative values considered time, effort (both physical and mental), skill, judgement, stress from iatrogenic risk and the complexity of the procedure.

These recommended values relate only to endoscopy procedures. They are not relative to any consultation or other procedure in the schedule of benefits. They are suggested for every physician group qualified to perform endoscopy. They are not tied to a dollar value.

In future, a technical component may need to be added to the fee. It should not change the relative 'professional' value.
COMPETENCE AND CREDENTIALLING FOR PHYSICIANS WHO PERFORM ENDOSCOPY

Endoscopy is a major tool for evaluating, diagnosing and treating patients with gastrointestinal tract disease. The procedures require not only technical skill, but also cognitive skills, and are best learned in an educational milieu with a skilled educator.

It is recognized that some endoscopic procedures are easy to learn, but there is more to endoscopy than guiding the endoscope.

The technical component is easiest to outline. In a formal training program graded responsibility occurs and, over time, there is less and less immediacy between teacher and student.

The skilled teacher recognizes when to intervene and when to allow the student more freedom.

There will never be complete agreement on the ideal number of procedures to be performed because of individual student skill. As a guideline, however, between 100 and 200 procedures for gastroscopy and at least 100 procedures for colonoscopy generally results in a safe candidate who should get better with experience.

All endoscopists should be trained to deal with therapeutic possibilities. 'Diagnostic endoscopy' should be a thing of the past. That is best for the patient and is costeffective, an important factor in these times of fiscal constraint.

Cognitive skills can be defined (eg, the indicators, risks) and measured by examination. Any physician performing endoscopy should know when it is or is not indicated, if it is safe and what to do with the information seen.

The ideal experience for learning endoscopy is in a recognized gastroenterology fellowship or surgical residency. A qualified endoscopist requires enhanced training. Training for physicians outside of these programs should include the same guidelines as for those in fellowship training. Patients will then always have a skilled operator to deal with endoscopic findings particularly if a therapeutic application is necessary.

A director of endoscopy should assess the candidate's skills in an ongoing program. A minimum number of procedures should be accomplished without assistance but with supervision. There should be a written test relating to endoscopy and gastrointestinal disease for those outside a regular training program.

Hospital credentials committees: Hospital credentials committees and hospital boards have an obligation to protect their patients. CAG guidelines should influence these decisions.

Deciding who should perform gastrointestinal endoscopy in a given hospital should be based on certified knowledge and training experience in the management of gastrointestinal disease as well as procedural competency. The same standards should also apply to all candidates.

It is recommended that credentialling boards withhold endoscopy privileges until those seeking privileges have been observed in clinical situations requiring demonstration of their skills. 
A proctor should observe each procedure, both diagnostic and therapeutic, and prepare a written report to the credentialling body. The credentialling board should ask the following questions.

- Does the hospital need the procedure?

- Can the individual do the job?

- Can the individual demonstrate competence?

Short courses in endoscopy: Short courses in endoscopy do not train physicians adequately. Cognitive skills and practical experience are limited. These poorly trained endoscopists are unacceptable as providers of basic endoscopy. Credentialling such physicians leads to poor patient care.

Fibreoptic sigmoidoscopy: Fibreoptic sigmoidoscopy may be performed in less elaborate facilities by physicians with less extensive training. If a rural hospital can show need for the procedure and will maintain the equipment, endoscopy units might consider establishing a modified program of instruction. A short 15 to $30 \mathrm{~cm}$ endoscope is recommended. Urban areas are unlikely to require family physicians to perform these procedures because trained specialists are available.

Competence and learning do not stop at the end of a training period. Maintenance of competence should be an ongoing educational process. Documentation of the continuing performance of endoscopists will help determine safety, effectiveness and efficiency, injecting objectivity into the recredentialling process.

Endoscopic competence: Gastrointestinal endoscopists should be well trained in the management of gastrointestinal disease as part of a training program in gastroenterology or surgery.

Endoscopy privileges should be considered only for applicants trained in an accredited program. Short courses are unacceptable as the sole training experience.

Endoscopy privileges should be granted for each separate procedure. Competence should be demonstrated to a proctor representing the credentialling body.

Endoscopy privileges should be periodically reviewed.

\section{INFECTION CONTROL IN ENDOSCOPY}

Endoscopes should be clean and free from infection.

Instances of infection with endoscopes are rare but obviously are of concern. The risks depend on the following:

- the instrument's exposure to microorganisms;

- cleaning; and

- disinfecting.

Dr David Fraser, microbiologist, was extremely helpful as a resource person at the consensus conference. The guidelines for cleaning were a natural extension of his expert opinions as well as a result of other published reports. In his review of the literature he encountered 67 cases of disease and two deaths related to infection from endoscopes. There have been no deaths since 1974. Twenty-four cases were from improper disinfection, 26 from a contaminated automatic washer, 12 of unknown cause and the remainder from contaminated air, water and biopsy channels. There was one case of hepatitis B, no cases of tuberculosis and no cases of human immunodeficiency virus (HIV).

A high level of disinfectant will result in a safe product (ie, a disinfected endoscope). Concern that exceptions exist centre around eradication of bacterial spores, Helicobacter pylori, pseudomonas, tuberculosis, hepatitis B and HIV.

Glutaraldehyde will eliminate all microorganisms except bacterial spores, and these spores don't cause disease when in contact with mucous membranes. Of interest, pathogenic spores are usually less resistant to glutaraldehyde than the spores used for laboratory testing of disinfectants. Clostridium difficile, of great clinical importance, is regularly killed on exposure to glutaraldehyde.

Where disinfection procedures are inadequate, $\boldsymbol{H}$ pylori can be transmitted. A thorough mechanical cleaning eradicates almost all $\mathrm{H}$ pylori (99\%) and glutaraldehyde kills the remainder.

Pseudomonas is of concern. It flourishes in warm, damp environments including sinks and water bottles. It is found in the throat $(0 \%$ to $7 \%)$, sputum $(2 \%)$ and stool $(3 \%$ to $24 \%)$. It is susceptible to glutaraldehyde.

Most reported pseudomonas infections occurred with ERCP. The endoscopes were likely not properly dried before overnight storage; the bacteria in the remaining water proliferated and were 'injected' into the biliary and pancreatic ducts causing sepsis. Drying the endoscopes and recleaning before use prevents this complication.

Mycobacterium tuberculosis is increasing in the community, an obvious concern. Glutaraldehyde is a widely accepted antimycobacterium agent. Studies have shown 2\% glutaraldehyde achieves a $99 \%$ reduction of $M$ tuberculosis in 10 mins.

Hepatitis B is common; the United States has about one million carriers. The virus is found in all body fluids. Only one case of hepatitis B transmission with an endoscope has been reported. There are 12 reports totalling 354 patients exposed to endoscopes used in hepatitis B antigen-positive patients and no passage of the virus occurred.

HIV is isolated in most body fluids although direct proof of transmission exists only with blood and semen. Cleaning in detergent effectively removes HIV from contaminated instruments (HIV transmission by endoscopes has never been reported).

Evidence shows that hepatitis B, HIV and most viruses are inhibited by $2 \%$ glutaraldehyde in 1 to 5 mins. The British Society of Gastroenterology recommends 4 mins of exposure to $2 \%$ glutaraldehyde in association with thorough cleansing to prevent viral transmission. In fact, that is their routine cleaning between patients and for precleaning.

The Working Party of the World Congress in Gastroenterology issued guidelines in 1990 emphasizing mechanical cleaning and a 10 min soak period in $2 \%$ glutaraldehyde. In 1991, the Association of Practitioners in Infection Control published their guidelines for selection and use of disinfec- 
tants. They recommended 20 mins of submersion time. The official Australian recommendation is a 2 min soak in $1 \%$ glutaraldehyde.

The most critical part of cleaning is mechanical. Good precleaning reduces the microbiological load by $99 \%$. If this is well done, 10 mins of disinfection is all that is necessary.

Enzymatic detergents are useful for cleaning; they help to clear out mechanical parts. A nonsurfactant soap works best although may be difficult to rinse out. Surfactants in soap have the potential to block the channels and should be avoided. The 14-day $2 \%$ glutaraldehyde solution is a nonsurfactant. The 28-day solution has surfactant.

Residents, Fellows and attendant staff must know how to clean the endoscopes.

Disinfectants: Two per cent glutaraldehyde is a very effective disinfectant, but a few points are worth remembering. The solution should not be diluted to be most effective. The channels should be flushed with glutaraldehyde, especially after mechanical washing. Over four to five days the 14-day glutaraldehyde solution may become diluted in washing machines, decreasing its effectiveness. Test kits are available to measure glutaraldehyde effectiveness. The disinfectant should be changed frequently depending, in part, on frequency of use and test results.

Glutaraldehyde is heavier than air, and chemical fumes drop rather than rise. Fume hoods may provide a false sense of security. Training in the use of glutaraldehyde helps keep levels low. Nonetheless, endoscopy staff have developed sensitivities to glutaraldehyde. An air rate of exchange 10 to 20 times/h is ideal in cleaning areas but older hospitals may not have this capacity. Glutaraldehyde levels may be measured for staff safety.

Glutaraldehyde is not a hazard to add to the water system. Run water and dilute it.

Automatic washers reduce glutaraldehyde fumes but have their own problems. Glutaraldehyde tends to become diluted and ineffective after four to five days. The machine needs cleaning every two weeks because it gets contaminated. Automatic washers have been known to miss cleaning steps. Their use tends to be slower than cleaning by technicians, and a busy endoscopy unit may need to buy more endoscopes.

Solutions such as glutaraldehyde are developed and tested on 'overkill'. An example is the case of M tuberculosis. Testing is measured on an endoscope contaminated with millions of bacteria. The endoscope is not washed. After 'disinfection' with glutaraldehyde that has been used for 14 days, the product label then can claim its effectiveness.

It is important to know that mechanical cleaning reduces the $\mathrm{M}$ tuberculosis count by $99.1 \%$. Ten minutes in glutaraldehyde reduces the count by $99.7 \%$. The additional 10 mins reduced the count by $0.03 \%$ ! Thus, standard guidelines of mechanical cleaning followed by 10 mins in $2 \%$ glutaraldehyde reduces $\mathrm{M}$ tuberculosis by at least $99.97 \%$. If $\mathrm{M}$ tuberculosis is a clinical worry, the extra 10 mins in glutaraldehyde is worthwhile.

Air and water can be a problem. Tap water frequently contains pseudomonas. Endoscopes that dry with water in the channels often are contaminated. Washing machines are contaminated in the same fashion. Compressed air has a high water content and can grow pseudomonas. If endoscopes are not completely dry, bacteria can reside in the channels. If forced air is not available then washing with $70 \%$ alcohol will complete dryness. Ventilated cupboards are a must.

Colonoscopes and gastroscopes with pseudomonas are not desirable but unlikely to cause disease. However, sepsis and pancreatitis has been caused by these organisms in sideview endoscopes at ERCP, a more invasive procedure. The safest approach is to resoak side-view endoscopes on the morning of their use. Institutions where pseudomonas may be a problem as suggested by regular bacterial assessment of endoscope channels should 'soak' the endoscopes preprocedure to overcome the risk of infection.

Water bottles, too, become contaminated with pseudomonas and need to be disinfected. A regular monitoring program to culture endoscopes, washing machines and other equipment should be in place. Cultures should never be positive.

Accessories for endoscopy designed to perforate the mucosa need to be sterile. A high level disinfectant is not acceptable. Gas sterilization is recommended.

Antibiotic prophylaxis: Antibiotic prophylaxis in endoscopy is controversial. Most guidelines are for prevention of bacterial endocarditis. Artificial knees and hips, vascular grafts and breast implants are rarely considered. Bacteremia occurs in fewer than $10 \%$ of procedures and, interestingly, is not any higher in colonoscopy and polypectomy than after a diagnostic sigmoidoscopy. Two procedures - sclerotherapy of esophageal varices and esophageal dilation - have a higher rate of bacterium. However, bacterial endocarditis after gastrointestinal endoscopy is very rare and antibiotic prophylaxis in this situation has never had a controlled trial. Thus, any recommendations are, at best, 'intuitive'.

\section{SEDATION AND MONITORING}

Sedation: Conscious sedation is the rule in gastrointestinal endoscopy. Exceptions do exist; a number of patients are endoscoped without sedation and a few are endoscoped under a general anesthetic.

The guiding principle for sedation is patient comfort and safety. The medication used may decrease anxiety or discomfort, reduce secretions or alter motility.

The required dose of drug should be estimated based on the patient's age, well-being, level of anxiety and prior use of medication. Administration by titration is preferable to bolus administration because it reduces the risk of overdosing and increases the safety of the procedure. Intravenous access is not mandatory but in many cases should be maintained throughout the procedure for administration of additional drug or emergency medications.

Benzodiazepines are the most commonly used agents in endoscopy. The most frequently prescribed drugs are diazepam and midazolam. The longer acting diazepam has a $24 \mathrm{~h}$ 
half-life and is less expensive. The newer midazolam has a half-life of 1.2 to $4 \mathrm{~h}$ but is more costly. Midazolam has greater potency because lower doses are required. No matter what drug is used for sedation, the minimal amount to achieve muscle relaxation and amnesia is desired. Relaxed patients are more cooperative and examination is easier.

For discomfort during endoscopic procedures, as can be the case with colonoscopy, a combination of benzodiazepines and opiates is useful. These drugs act synergistically with side effects of respiratory and cardiovascular depression so the endoscopic team needs to be alert to these possible complications.

Reversal agents for both opiates (naloxone hydrochloride) and benzodiazepines (flumazenil) are available and should be present in the endoscopy suite as emergency drugs. Monitoring: Monitoring a patient during endoscopy improves the outcome by decreasing the risks of sedation and of the procedure. Early detection of patient distress will improve safety. Suctioning, oxygen, use of light sedation, use of narcotic or benzodiazepine antagonists, stopping the procedure and changing the postprocedure follow-up care may all improve safety.

The best monitors of the patient's safety are the physicians and skilled assistants working as a team. Nothing replaces clinical assessment. The endoscopist should assess the complexity of the monitoring required for each patient's perceived needs. Most endoscopy procedures can be managed with one skilled assistant. More complex problems are best handled by two and occasionally more qualified personnel. Off-hours endoscopy, nights and weekends require equally skilled assistants because the patients are usually emergencies and seriously ill.

Standard clinical monitoring should include measuring heart rate, blood pressure and respiratory rate before sedation, during the procedure and immediately postprocedure as well as before discharge from the recovery area. A clinical assistant should be present throughout the procedure. The record should include the measurements and observations of any problems during the procedure.

The patient's tolerance to the procedure, including events such as vomiting and respiratory distress, should be noted and management documented.

Oximetry is useful but does not replace clinical observation. However, oxygen saturation is best detected by an oximeter. Decrease in oxygen saturation usually carries little clinical risk, but does on some occasions. Over half the serious adverse reactions to endoscopy are cardiopulmonary. Thus, it makes use of oximetry, a rational recommendation in all cases of conscious sedation.

Significant oxygen desaturation, when detected early, can be treated with increased ventilation, supplemental oxygen therapy, narcotic or benzodiazepine reversal, or termination of the procedure. Most sedation-related hypoxia can be prevented by low flow oxygen throughout the endoscopic procedure.

When monitoring devices are limited, specific situations for oximetry include prolonged procedures, procedures using a large upper endoscope, procedures in a darkened room, procedures requiring more than light sedation, and procedures performed on a patient who is elderly or has serious medical disease.

If a patient is at risk for cardiac arrhythmias, electrocardiogram (ECG) monitors should be used during endoscopy. ECG abnormalities are most common in the elderly and in patients with heart and lung disease.

Postendoscopy: After the procedure patients are still at risk for complications and thus monitoring is required until the patient is alert, speaking clearly, able to sit up and has stable vital signs.

\section{GUIDELINES FOR QUALITY IN GASTROINTESTINAL ENDOSCOPY}

Standards of care are guidelines based on literature review and the consensus of wise men. The phrase 'standards of care' in a legal sense defines the duty or responsibility that, in our case, the endoscopist must fulfil in care of any patient.

Standards of care, according to the American Joint Commission on Accreditation of Health Organizations, must include a written plan that describes the programs, objectives, scope and mechanism for overseeing the effectiveness of monitoring, evaluation and problem-solving arbitration.

The endoscopist must be trained and credentialed, and maintain and improve endoscopic skills.

Patients must be informed about the procedure. The key to this is disclosure of information. The patient must be aware of options, risks and benefits. A record of the consent is important.

Exceptions to the need for a patient's consent include the following:

- emergency;

- incompetence;

- therapeutic privilege; or

- waiver.

A two-physician consent form should be available to cover emergencies and/or the incompetent patient. Therapeutic privilege implies that disclosure of the endoscopic procedure will harm the patient but this is most unlikely. A patient may elect not to be informed or to waive rights about being informed about a procedure. Endoscopists should document these situations and obtain a two-physician consent form.

The endoscopy unit should have established guidelines. The ancillary personnel need proper training in assisting at endoscopy, and recognizing and dealing with complications and problems, including cardiopulmonary resuscitation procedures. Equipment for resuscitation should be at hand.

The unit should function with well maintained equipment. It is cost-effective to repair and monitor equipment. Cleaning endoscopes requires standard achievable results.

Areas should be designed for preparation, apart from the endoscopy suite and recovery areas. 
Endoscopy units need instrument cabinets that are well aerated for proper storage and maintenance of endoscopes.

There should be an adequate storage area for drugs, supplies and equipment.

Documentation of the patient's identity, indications for the endoscopy, procedural record and findings is mandatory. A daily log of the unit's activities should be kept.

Procedural reports and a record of the patient's association with the endoscopy unit should be maintained. This is best entered into a computer database. The information can be an index for quality improvement review. These data may identify problems relating to the endoscopy unit's functioning. Delays in scheduling, equipment failure, equipment needs and personnel requirements may become apparent and result in needed improvement.

The literature has several lists of appropriate indications for endoscopy. These lists are part of the standards of care that each endoscopy unit should develop.

The unit's documentation should be regularly reviewed to improve the quality of care. Such an approach has shown a reduction in unnecessary procedures. A peer review process is often needed to motivate a unit to use proper documentation.

Each endoscopic unit should maintain a record of all procedures and the endoscopist's performance. Complications should be recorded. These data, such as rates of incomplete examinations and complications, should be useful for improving the quality of endoscopic care.

Support personnel for an endoscopy unit are very important. They must be helped to develop their skills with onthe-job supervision. Enrolment in a national organization is encouraged.

An endoscopy unit does not need to be in a hospital. Out of hospital facilities should adhere to the same standards as are followed in hospitals. Consideration should be given to the availability of emergency transport when deciding upon a unit's location.

\section{QUALITY}

There is no simple definition of quality. The purpose of standards and guidelines is to institute a more appropriate use of gastrointestinal endoscopy resulting in improved patient care. Improvement in quality is a natural consequence of standards. Care can be evaluated, and stronger aspects lauded and weaker aspects brought to attention for possible improvement. The information collected can allow objective assessment of mortality, adverse occurrences or utilization.

Quality improvement in endoscopy directs attention to the procedure and the endoscopic unit. Endoscopic practice should be reviewed regularly by a committee of endoscopists and others interested in quality improvement. The group should report to the appropriate department.

Reviews and actions of the committee should improve patient care. The data obtained can be considered for recredentialling recommendations to department chiefs and credentialling committees. Endoscopic privileges should be based on performance as well as continuing education. Patterns of performance and individual cases should be examined.

The endoscopy committee's review of procedures may include the following:

- appropriate indications and number of procedures;

- absence of contraindications;

- technical performance (colonoscopy to cecum, successful polypectomy, cannulation of desired ducts at ERCP);

- complications, including need for oxygen, assisted respiration, etc; and

- correlation of results with desired outcome (ie, data for outcome review).

Physicians could also be reviewed on cases outside a predetermined 'threshold' such as:

- inability to complete an examination $80 \%$ of the time;

- incident reports;

- random sampling; or

- fewer than a desired number of procedures to maintain skill.

Records indicating a problem should be reviewed by the committee.

Comprehensive documentation would provide information for in-depth evaluation of specific aspects of patient care related to endoscopy. Examples include the following:

- length of stay;

- transfusion requirements for endoscopically treated gastrointestinal bleeding;

- incidents of complications such as pancreatitis postERCP; or

- adequacy of patient preparation before colonoscopy.

The number of procedures, indications, results and complications should be available to the chief of service or committee responsible for granting privileges. Complications should be discussed at a periodic conference as a mechanism for review of performance and as an educational device. These suggestions offer a means, at times imperfect, to monitor ongoing competence of technical skills.

ACKNOWLEDGEMENTS: The committee recognizes the American Society for Gastrointestinal Endoscopy and their numerous excellent published guides on endoscopy. 


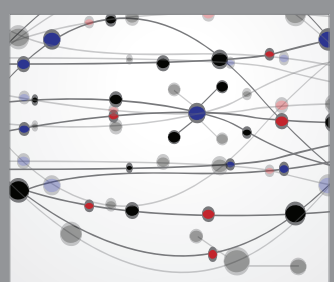

The Scientific World Journal
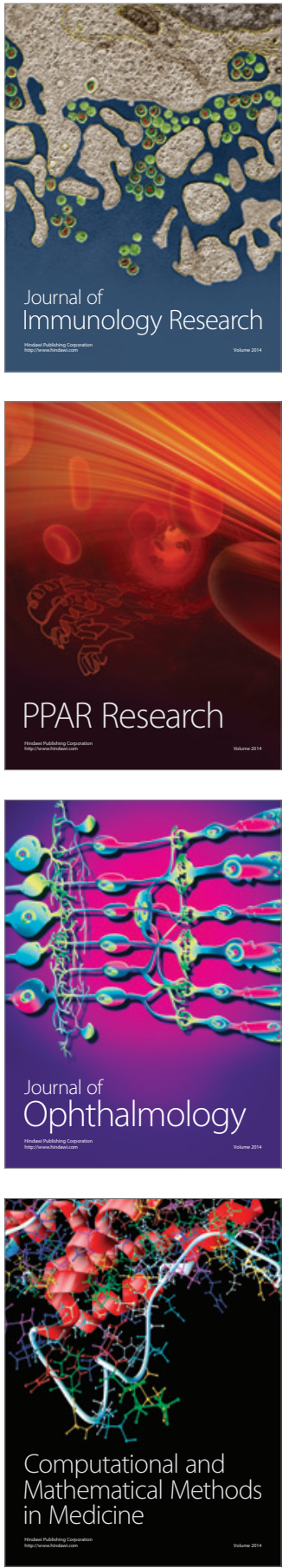

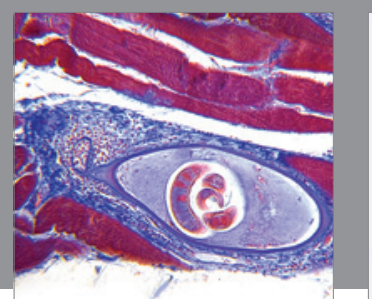

Gastroenterology Research and Practice

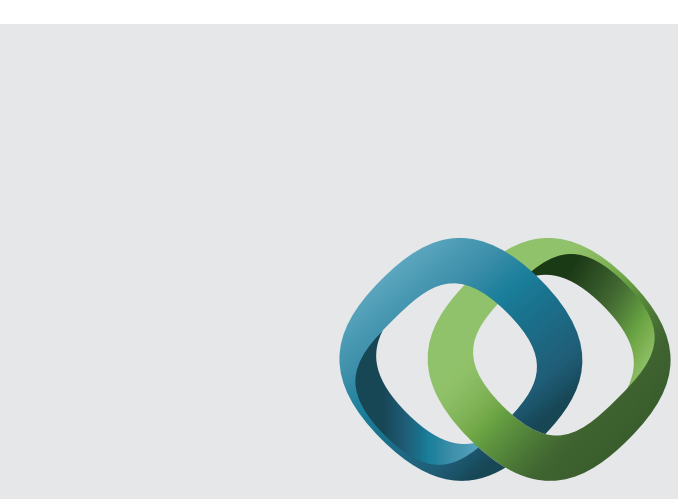

\section{Hindawi}

Submit your manuscripts at

http://www.hindawi.com
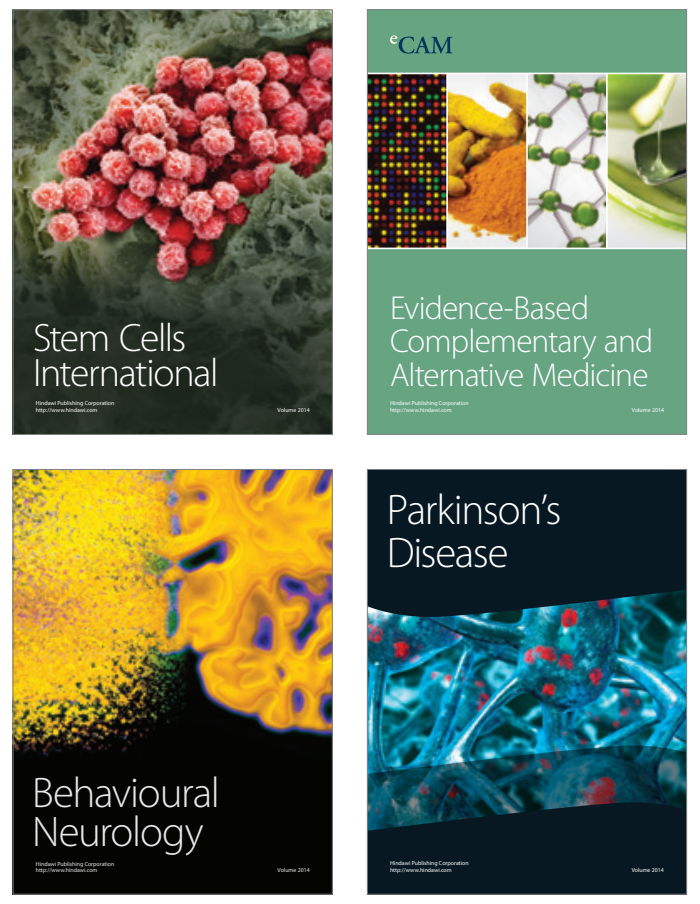
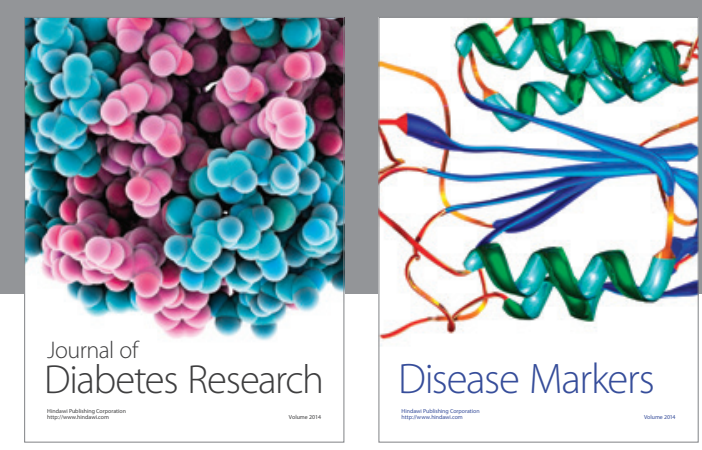

Disease Markers
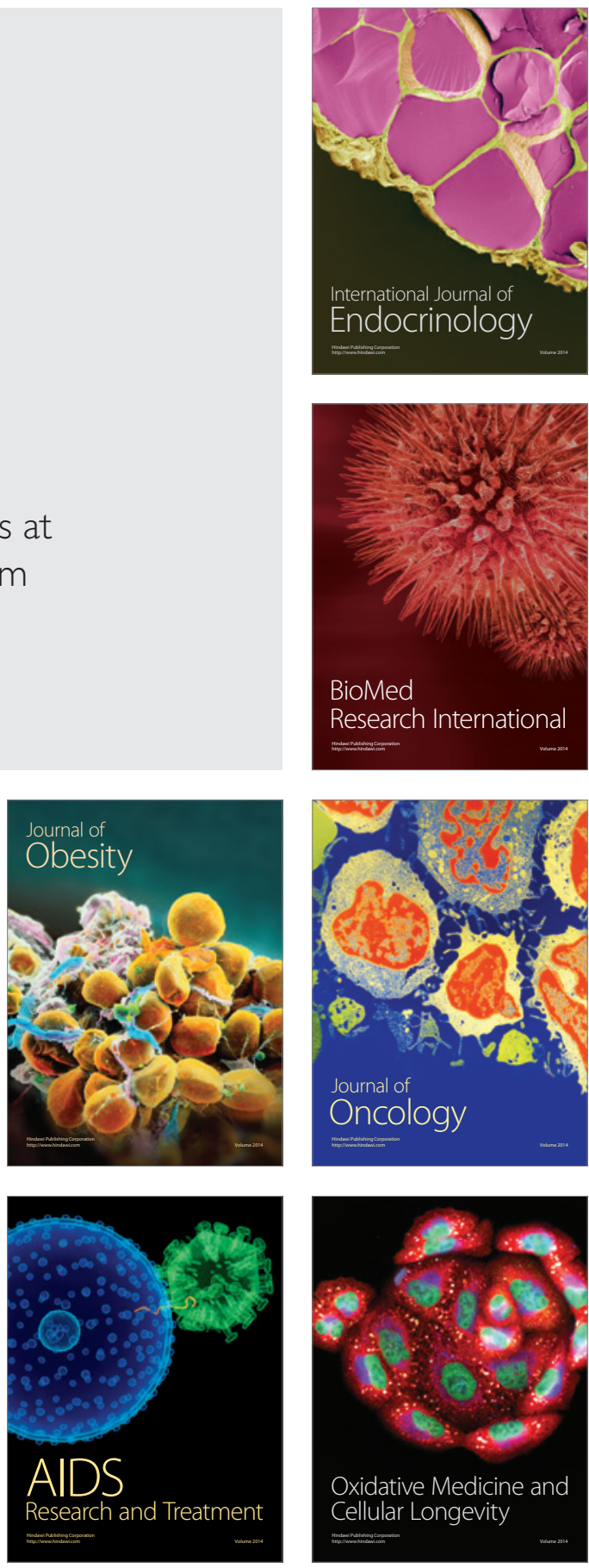